Jan TRNA

https://orcid.org/0000-0003-3138-9736

Masarykova univerzita Brno

\title{
Weinen und Lachen als tragende Säulen der Schweiz-Kritik im Theaterstück Zwanzigtausend Seiten von Lukas Bärfuss
}

\section{Crying and laughing as the pillars of criticism of Switzerland in the play Zwanzigtausend Seiten by Lukas Bärfuss}

\begin{abstract}
The focus of this article is the little-explored play Zwanzigtausend Seiten by the Swiss playwright Lukas Bärfuss. The criticism of Swiss society is presented under the focus of the emotional manifestations of laughter and crying. The focus is on two main protagonists, Wüthrich and Tony. One weeps constantly in his hiding place over the negative attitude of Swiss society to its behavior during World War II. The other tries to make the serious allegations public through an amusing TV show. The following research questions are examined in this regard: Are the main characters Wüthrich and Tony to be understood as complementary oppositions, each of which can be equated with crying and laughing? For the same reason, have their efforts to criticize Swiss coping with the past concerning the atrocities of the Second World War failed? What position does society take regarding these two individuals, and what role do crying and laughter play in this? Keywords: laughter, crying, Zwanzigtausend Seiten, Lukas Bärfuss, empathy, criticism of Switzerland.
\end{abstract}

Der vorliegende Aufsatz setzt sich mit dem durch die Schweiz-Kritik geprägten Theaterstück Zwanzigtausend Seiten ${ }^{1}$ und den Funktionen von Wei-

1 Das Theaterstück Zwanzigtausend Seiten wurde in mehreren Besprechungen auf sein gesellschaftskritisches Potenzial hin befragt. Vgl. etwa Beat Mazenauer, Elend ist keine Stil- 
nen und Lachen auseinander, die als emotionelle Grenzwerte betrachtet und mit Blick auf den dramatischen Text untersucht werden sollen. ${ }^{2}$ Den Dramen von Lukas Bärfuss wird attestiert, dass sie ihre Figuren ausweglosen Situationen ausliefern. Nicht anders verhält es sich auch in diesem Stück. Lachen und Weinen, so eine These dieses Beitrags, können als Reaktionen der Figuren auf eine solche Ausweglosigkeit erachtet werden. Die Konfrontation mit gesellschaftlichen Zwängen und die Unmöglichkeit, auf einen vorherrschenden Diskurs Einfluss zu nehmen, treiben die Figuren zu höchst persönlichen emotionellen Ausbrüchen, die im Folgenden analysiert werden.

Die Schweiz-Kritik in Zwanzigtausend Seiten baut sich auf der Trostlosigkeit von zwei Individuen auf, die darunter leiden, von der Gesellschaft nicht ernst genommen zu werden. Dabei haben sie aber äußerst ernsthafte Inhalte zu berichten. Sie setzen sich nämlich mit der Rolle der Schweiz im Zweiten Weltkrieg auseinander und wollen, dass auch die breite Öffentlichkeit an dieser Auseinandersetzung teilhaben kann. ${ }^{3}$ Da sie, wie gesagt, auf Unverständnis stoßen, fällt ihre Reaktion auf zweierlei Weise aus: Während Wüthrich in seinem Unterschlupf über die ablehnende Haltung der Schweizer Ge-

frage, https://nachtkritik.de/index.php?option=com_content\&view=article\&id=6546: zwanzigtausend-seiten-ua-lars-ole-walburgs-inszenierung-von-lukas-baerfuss-neuemstueck-am-schauspielhaus-zuerich-fragt-wieviel-verantwortung-wir-fuer-die-vergangenheit-tragen-wollen\&catid=38:die-nachtkritik\&Itemid=40, Claudio Steiger, Geschichte ohne Schüler, https://www.nzz.ch/geschichte_ohne_schueler-1.14771452; Lisa Letnansky, Wozu das ganze Spektakel?, https://www.nahaufnahmen.ch/2012/02/04/lukas-barfusszwanzigtausend-seiten-schiffbaubox-zurich/. Bis jetzt ist aber ein wissenschaftlicher Beitrag ausgeblieben, der sich mit den Emotionen in diesem Stück beschäftigt und sie als wesentliche Merkmale der Schweiz-Kritik betrachtet.

2 Eine der grundlegendsten Publikationen, die diese Problematik sogar im Titel führt, stellt Helmut Plessners Schrift Lachen und Weinen. Eine Untersuchung der Grenzen menschlichen Verhaltens dar. Er versteht diese beiden biologischen Reaktionen als Phänomene, die für den Menschen typisch sind und auf eine Krisenlage zurückzuführen sind, in der er sich befindet. Vgl. Helmut Plessner, Lachen und Weinen. Eine Untersuchung der Grenzen menschlichen Verhaltens (München: Leo Lehnen/Francke 1950). Auf dieses Buch geht in seinem Aufsatz Joachim Fischer ein und erläutert Plessners philosophisch-anthropologischen Ansatz. Vgl. Joachim Fischer, „Helmut Plessner: „Lachen und Weinen. Eine Untersuchung nach den Grenzen des menschlichen Verhaltens". In Hauptwerke der Emotionssoziologie, hrsg. v. Konstanze Senge u. a. (Wiesbaden: Springer, 2013), 274-279.

3 Ein Bericht von der Schweiz, dem Nationalsozialismus und dem Zweiten Weltkrieg ist 2002 tatsächlich entstanden. Vgl. Die Schweiz, der Nationalsozialismus und der Zweite Weltkrieg: Schlussbericht, ed. Jean-François Bergier et al. (Zürich 2002), https://www.researchgate.net/publication/278749844_Die_Schweiz_der_Nationalsozialismus_und_der_

Zweite_Weltkrieg_Schlussbericht. Der Name des Hauptverfassers weist sogar Ähnlichkeiten mit dem Namen der fiktiven Figur auf. Es ist jedoch nicht das Ziel des vorliegenden Beitrags, intertextuellen Verweisen nachzuspüren. Lukas Bärfuss stellt in seinen Dramen Themen in den Raum, die in der Schweizer Gesellschaft eine hohe Brisanz besitzen. Weitere ähnliche Beispiele kann man etwa bei der Problematik der Sterbehilfe finden. 
sellschaft zu ihrem eigenen Verhalten im Zweiten Weltkrieg unentwegt weint, versucht Tony, die ernsthaften Anschuldigungen mittels einer amüsanten TV-Sendung publik zu machen. Man flüchtet sich also entweder ins Weinen oder zieht sich ins Lächerliche zurück, um auf sich und die eigene Botschaft aufmerksam zu machen. Folgenden Forschungsfragen wird diesbezüglich nachgegangen: Sind die Hauptfiguren Wüthrich und Tony als komplementäre Oppositionen aufzufassen, die jeweils mit Weinen und Lachen gleichzusetzen sind? Sind ihre Bemühungen, eine Kritik an der Schweizer Vergangenheitsbewältigung hinsichtlich der Gräuel im Zweiten Weltkrieg auszuüben, aus dem gleichen Grund gescheitert? Welche Position bezieht die Gesellschaft zu diesen zwei Individuen und welche Rolle nehmen Weinen und Lachen dabei ein?

\section{Weinen und Lachen aus biologischer und gesellschaftlicher Sicht}

Begibt man sich auf die Suche nach der herkömmlichen Auffassung von Weinen und Lachen, so stellt man fest, dass sie viel älter als etwa das Tragische/Komische sind, ganz zu schweigen vom Humor. ${ }^{4}$ Die grundsätzliche Frage nach der Funktion von Lachen und Weinen beantwortet Vallori Rasini wie folgt:

Was stellen Lachen und Weinen dar? Es sind Entäußerungen von unsymbolischer (im Gegensatz zu den Gesten beispielsweise), eruptiver und unkontrollierter Art (im Gegensatz zu sprachlichen Äußerungen), in denen der Mensch entgleist, indem er es dem Körper überantwortet, auf eine existenziale Situation besonderer Schwierigkeit zu antworten. ${ }^{5}$

Weinen und Lachen stehen somit für körperliche Reaktionen, die kognitiv nicht in den Griff zu bekommen sind. Da dies aber mit der Vorstellung kollidiert, Menschen seien Lebewesen, die in den allermeisten Fällen ihre Emotionen unter Kontrolle haben, wird ständig auf ihren grenzwertigen Charakter hingewiesen. Es handelt sich also um einen unfreiwilligen Verzicht, eigene Gedanken bzw. Bedürfnisse auf gestische und/oder sprachliche Art und Weise zum Ausdruck zu bringen. Wird man mit einer Situation kon-

4 Auf Humor und seinen Bezug zum Lachen geht Radim Brázda in seiner dem Philosophen Odo Marquard gewidmeten Abhandlung ein. Vgl. Radim Brázda, „Odo Marquard, Humor, Vernunft und Lachen“, Pro-Fil 16, Nr. 2 (Brno: Masarykova univerzita, 2015): 51-68.

5 Vallori Rasini, „Menschlicher Ausdruck und Grenzverwirklichung. Lachen und Weinen, " in Expressivität und Stil. Helmuth Plessners Sinnes- und Ausdrucksphilosophie, hrsg. v. Bruno Accarino und Matthias Schlossberger, «Internationales Jahrbuch für Philosophische Anthropologie», Bd. 1 (Berlin: Akademie, 2008): 204. 
frontiert, der man - aus welchem Grund auch immer - weder emotional noch kognitiv gewachsen ist, kommt einem die Kontrolle über die eigenen Emotionen abhanden und Wein- oder Lachreaktionen stellen sich ein.

Begegnet man jedoch einer weinenden oder lachenden Person - und damit kommen wir nun zum (teilweise) gesellschaftlichen Aspekt dieser Problematik - passiert etwas, was man nur bedingt zu kontrollieren vermag. Der menschliche Körper reagiert nämlich auf seinen sich in einem solchen $\mathrm{Zu}$ stand befindenden Gattungsgenossen mit Empathie. ${ }^{6}$ Man versucht, sich in die Lage des Gegenübers hineinzuversetzen und verspürt die triebbedingte Nötigung, den Grund für jene Wein- oder Lachreaktion zu ermitteln. Die Fachliteratur macht an dieser Stelle die Spiegelneurone dafür verantwortlich, dass die Gefühlsäußerungen anderer einem nicht gleichgültig bleiben, sondern dass sie zu einer eingehenden Auseinandersetzung anregen.

\section{Das Tragische und Komische und ihr Bezug zum Weinen und Lachen}

Im Gegensatz zu Weinen und Lachen, die dem menschlichen Willen nicht unterliegen, versteht man das Tragische und das Komische als durchdachte Konzepte. Die Handhabung und Instrumentalisierung dieser reflexiven Phänomene spielt für die Funktion des Tragischen und Komischen eine brisante Rolle. Für die weitere Textanalyse ist vor allem das Komische von Bedeutung und wird daher entsprechend akzentuiert.

Das Adjektiv komisch geht zurück aufs Lateinische und Griechische und bedeutet so viel wie „1. zum Lachen reizend, belustigend; 2. eigenartig, sonderbar. ${ }^{\text {7 }}$ Das Komische versteht sich also als ein Mechanismus, der auf sprachlichen und nichtsprachlichen Handlungen (etwa Gesten) beruht und bei anderen Personen Lachen herbeiführen soll. Es handelt sich somit um eine bewusste Handlung, die beim Publikum eine unbewusste, durch Verstand und Vernunft nicht kontrollierbare Reaktion wecken soll. Tragisch wird dagegen mit „,schicksalhaft, erschütternd und ergreifend“" ${ }^{8}$ wiedergegeben. Im Gegensatz zu komisch findet man an erster Stelle keine Erwähnung des Weinens vor, nämlich etwa zum Weinen reizend. Obwohl man Lachen

6 Empathie wird hier als die Fähigkeit aufgefasst, sich in eine andere Rolle hineinzuversetzen, und diesbezüglich als die Bereitschaft, das eigene Verhalten mit Blick auf die Wünsche und Bedürfnisse des Gegenübers modifizieren zu wollen. Zur Begriffserklärung Empathie vgl. etwa Empathie und Erzählung, hrsg. v. Claudia Breger u. a. (Freiburg i. Br./Berlin/ Wien: Rombach Litterae, 2010), 7-20.

7 Duden. Deutsches Universalwörterbuch (8. Auflage, Berlin: Duden Verlag, 2015), 1027.

8 Duden. Deutsches Universalwörterbuch, 1778. 
und Weinen als Grenzerscheinung menschlichen emotionellen Verhaltens versteht, nimmt das Lachen im Komischen eine zentrale Position ein, während das Weinen offensichtlich als unerwünscht angesehen wird. Diese Feststellung mag von daher rühren, dass das Weinen vor allem bei Erwachsenen sehr ernsthafte Gründe haben kann, die eher nicht angesprochen werden sollen. Man möchte ja emphatisch vermeiden, versehentlich ein Tabuthema zu eröffnen und somit der betroffenen Person nahezutreten.

Ingrid Hentschel attestiert dem Komischen eine zunehmend bedeutende Rolle am zeitgenössischen Theater. Dabei führt sie einen weiteren wichtigen Begriff - die Lachkultur -ein, der für die weiteren Ausführungen von großer Relevanz sein wird. Lachkultur ist ihrer Meinung nach bei Versuchen, das Agieren der Gesellschaft besser zu verstehen, besonders wichtig, denn dies, „[w] $[$ orüber eine Gesellschaft lacht - und wie sie lacht, ihre Lachkultur - ist immer auch Indiz für ihre gegenwärtige Verfasstheit". ${ }^{9}$ Die nachfolgende Textanalyse konzentriert sich somit auf jenes Worüber, d. h. auf die Frage, warum die Gesellschaft über das eine lacht, über das andere jedoch nicht. Was versteht sie also als Teil der Unterhaltung und was möchte sie besser unberücksichtigt lassen, weil es nicht mehr lustig, komisch oder lachhaft ist? Lachkultur versteht sich als eine institutionalisierte Herangehensweise an das Lachen, bei der zu entscheiden ist, welches Format heranzuziehen ist, um das Publikum zum Lachen zu bringen.

\section{„Ich will kein Niemand sein, lieber der Jemand, der weint." 10 Weinen als Schweiz-Kritik}

Das 2012 am Schauspielhaus Zürich uraufgeführte und im selben Jahr in Druckform herausgebrachte Theaterstück Zwanzigtausend Seiten ist bislang von der Forschung wenig beachtet worden. Die Hauptfigur Tony ist ein junger, arbeitsscheuer und ungebildeter Mann mit krimineller Vergangenheit, der sich am liebsten im gleichgesinnten Freundeskreis dem Alkoholrausch hingibt oder drittklassige Filme anschaut. Er lebt bei seiner Freundin Lisa, von der er finanziell abhängig ist und die ihm Geld für seine „Hobbys“ zukommen lässt.

Nach einem Unfall, bei dem Tony eine Kiste voller Bücher auf den Kopf fällt, wird er in eine psychiatrische Klinik eingeliefert, weil er zwar körperlich unversehrt geblieben ist, aber seitdem behauptet, den Inhalt der Bücher

9 Ingrid Hentschel, „Spielen mit der Performanz. Transformationen des Komischen im Gegenwarttheater", Maske und Kothurn 54, Nr. 4 (2005): 214.

10 Lukas Bärfuss, „Zwanzigtausend Seiten,“ in Lukas Bärfuss, Malaga, Parzifal, Zwanzigtausend Seiten. Stücke (Wallstein Verlag, Göttingen 2012), 157. 
vollständig im Gedächtnis gespeichert zu haben. Es handelt sich dabei um 25 Bände - insgesamt zwanzigtausend Seiten - die sich mit der Rolle der Schweiz im Zweiten Weltkrieg auseinandersetzen. Abgedruckt wurden darin auch Briefe von denjenigen, die vor dem NS-Regime in die Schweiz flüchteten, jedoch abgewiesen und somit zum fast sicheren Tod in KZs verurteilt wurden. Zudem wurden in den Bänden Fälle von wohlhabenden Nazi-Größen und Kriegsverbrechern geschildert, die in der Schweiz nach dem Kriegsende Zuflucht fanden und als angesehene Bürger ihren Lebensabend in der Eidgenossenschaft verbringen konnten.

Als Tony einsieht, dass er sowohl von der Chefärztin Gosbor wie auch von seiner Freundin Lisa für geisteskrank gehalten wird, flieht er aus der Klinik und sucht den Herausgeber der Bücher auf, einen gewissen Jean Michel Blonay. Dennoch kommt kein persönliches Gespräch mit ihm zustande, sondern nur mit Professor Blonays Assistentin Lorena, die seine Meinungen wortgetreu zu zitieren weiß. Blonay sei, nachdem er beauftragt wurde, einen offiziellen Bericht über das Verhalten der Schweiz im Zweiten Weltkrieg zu erarbeiten, öffentlich angegriffen und bloßgestellt worden, was seine Assistentin folgendermaßen kommentiert:

Rückgrat, so der Professor, sei hierzulande Synonym für Lebensuntüchtigkeit. [...] [Z]wangsläufig [trete] die Prinzipienlosigkeit, die Speichelleckerei, die Korruption und die Heimtücke als Grundpfeiler der hiesigen Identität zutage. ${ }^{11}$

Erbittert warf also Blonay selbst die Kiste mit den Büchern aus dem Fester, unter dem sich unglücklicherweise gerade Tony befand. Seitdem beschäftigt sich Blonay mit der Klassifizierung verschiedener Knöpfe - nach Farbe, Form, Zahl der Löcher, etc. - was er, im Vergleich zu dem, was er vorher getan hat, für eine durchaus plausible Tätigkeit hält.

Tony wird von nun an von den Geschichten in seinem Kopf geplagt, und er kann seinen neu gefundenen Gerechtigkeitssinn nicht mehr loswerden. Der Verzweiflung nahe macht er einen Sonderling namens Wüthrich ausfindig, der jene Bücher vor Monaten entliehen hat und auf jegliche Mahnungen der Bibliothek nicht reagiert. In einem vermoderten Unterschlupf im Wald begegnet Tony dem dahinsiechenden Mann, der sich in seinem verfaulten Zuhause einen „Altar der Schande“ aus den Büchern errichtet hat:

WÜTHRICH Schande, ewige Schande. Er weint.

TONY Warum weinen Sie.

WÜTHRICH Weil du nicht weinst. Weil keiner weint. [...] Ich weine über die Ignoranz, über die Dummheit, die Gewinnsucht, die Herzlosigkeit, die Selbstsucht, die Verleugnung [...] über die abgewandten Blicke und über das Wachs in den Ohren der

11 Bärfuss, „Zwanzigtausend Seiten“, 148. 
Menschheit. Ich weine über die Unschuldigen, die ermordet wurden, ermordet werden und ermordet werden werden. ${ }^{12}$

Wüthrich, einst ein angesehener Journalist mit tadellosem Ruf, nun aber ein gebrochener Mann, sieht sich der Lage aussichtslos ausgeliefert. Auch die Namensgebung mag als Hinweis auf seine geistige Verfassung angesehen werden, denn er verspürt unentwegt eine hemmungslose Wut gegenüber der ganzen Gesellschaft. Er beklagt sich über die Teilnahmslosigkeit seiner Mitmenschen, die sich der geschichtlichen Wahrheit nicht stellen wollen. Den Mangel an Empathie in der Gesellschaft wiegt er dadurch auf, dass er den Bann des Weinens auf sich nimmt. Weinen hat für ihn etwas Kathartisches und Auserwähltes zugleich: „Ich trage, was keiner trägt. Wenn keiner schuldig ist für nichts, dann bin ich schuldig für alles."13 Es sind also nicht nur Trauer und Ausweglosigkeit, die ihn zum Weinen bringen, es ist auch die Schuld seines Vaterlands, die er mit niemandem teilen kann, d. h. die nichtexistenten Schuldgefühle der Schweizer gegenüber den Opfern des Kriegsgeschehens. Da Wüthrich mit seinen Erkenntnissen an die Öffentlichkeit ging und kläglich scheiterte - genauso wie Professor Blonay -, zog er sich an einen Ort zurück, wo er nicht ausgelacht wird. Dort kämpft er mit den Dämonen der Vergangenheit, die seine eigenen geworden sind.

Plessner versteht Lachen und Weinen als Reaktionen, die einen individuellen Charakter haben. Auch hier sieht sich die Figur Wüthrich persönlich betroffen. Möchte man aber seinem Weinen auf den Grund gehen, stellt man fest, dass der Auslöser auf einen nicht individuellen, sondern gesellschaftlichen Charakter zurückzuführen ist. „Immer wieder sind Bärfuss' Figuren ausweglosen Situationen ausgesetzt, in denen ihr Selbstbild, ihre Wünsche, ihr Begehren und ihre soziale Rolle in Konflikt geraten. "14 In gewissem Sinne lässt sich das Verhalten von Wüthrich als eine ad absurdum geführte Empathie-Reaktion verstehen, die durch Weinen zum Ausdruck gebracht wird. Das Verhalten der Mehrheitsgesellschaft, die sich keinen konkreten Individuen zuschreiben lässt, wirkt sich auf den psychischen Zustand eines bestimmten Individuums aus. Die oben erwähnte Ausweglosigkeit, die Bärfuss'schen Dramen attestiert wird, kommt bei Wüthrich wiederum zum Tragen. Abgeschieden von seinen Mitmenschen, die auf seine Botschaft nicht hören wollten, weint er verkrochen in einem Wald-Unterschlupf, versteckt vor den Blicken anderer. Es ist somit ausgeschlossen, dass sein Weinen jemanden rühren oder gar zum Nachdenken anregen könnte. Nicht einmal To-

\footnotetext{
Bärfuss, „Zwanzigtausend Seiten“, 156.

Bärfuss, „Zwanzigtausend Seiten“, 157.

14 Handlungsmuster der Gegenwart. Beiträge zum Werk von Lukas Bärfuss, hrsg. v. Marie Gunreben u. a. (Würzburg: Königshausen und Neumann, 2017), Umschlagtext nicht nummeriert.
} 
nys Einfühlungsvermögen wird beim Anblick des weinenden Wüthrichs ansatzweise aktiviert.

\section{„Ich werde nicht mit diesem Elefanten auf dem Kopf - das ist albern." ${ }^{15}$ Lachen als Schweiz-Kritik}

Tony lehnt es resolut ab, eine solche Einstellung mit Wüthrich zu teilen, und entscheidet sich, in der Öffentlichkeit publik zu machen, was während und nach dem Weltkrieg in der Schweiz vor sich ging. Doch sein Versuch, über einen Auftritt in einer Radiosendung gesellschaftliche Aufmerksamkeit zu erlangen, scheitert, da keiner auf seine Botschaft reagiert. In einem ernsthaften Format zu den Mitmenschen zu sprechen, erweist sich somit für seine Intention ungeeignet. Das Radio ist offensichtlich nicht mehr das Medium, mit dem eine breite Zuhörerschaft erreicht werden kann. Darüber hinaus scheint auch die Art und Weise, wie Tony an die Öffentlichkeit tritt, nicht die Richtige. So zumindest stellt es John dar, ein Künstleragent, der anscheinend der einzige Rezipient dieser Sendung war und der nun Tony anspricht und ihm vorschlägt, an einem Talent-Wettbewerb teilzunehmen, um die Aufmerksamkeit der Menschen auf seine Botschaft zu lenken. Tony solle einfach als Gedächtniskünstler agieren. Nicht das Ernsthafte, sondern das spielerisch Komische sollte nun der richtige Weg dafür sein, ernstzunehmende geschichtsträchtige Inhalte den breiten Massen übermitteln zu können. Damit gelangt Tony in das Milieu des Reality-TV-Formats, das den Forschungsgegenstand zahlreicher Studien bildet. ${ }^{16}$ Menschliche Würde stellt in diesem Format oft keine ernstzunehmende Kategorie dar. Man wird Teil eines Spektakels, wo der Spaß der Masse in den Vordergrund rückt, anders als die eigentliche Botschaft.

Im Verlauf der Handlung entpuppt sich John jedoch als Betrüger, der ständig versucht, Tony in die Rolle eines „Showmasters“ hineinzumanövrieren, und damit das Preisgeld von einer Million für sich zu beanspruchen. Dies beginnt Tony spätestens zu dem Zeitpunkt klarzuwerden, als er von John gezwungen wird, mit einem Brainforce-Plüschelefanten auf dem Kopf aufzutreten, einem Werbegegenstand, der ein Nahrungssupplement für die legale Steigerung der Gedächtniskapazität bewirbt. Trotz anfänglicher Abneigung ordnet sich Tony seinem Manager John unter und tritt im Finale gegen eine singende Busfahrerin an. Tony verliert seinen Wert als Mensch und seine

15 Handlungsmuster der Gegenwart. Beiträge zum Werk von Lukas Bärfuss, 183.

16 Vgl. dazu etwa Thomas Tanja, „,Mensch, burnen musst Du!' Castingshows als Werkstatt des neoliberalen Subjekts“, Zeitschrift für Politische Psychologie 12, Nr. 1-2 (2004): 191-208. 
Botschaft wird in Mitleidenschaft gezogen. Er befindet sich in einer Lage, in der er sich für Werbezwecke lächerlich macht und in einem zweifelhaften Duell antritt.

Performative Reality-TV-Formate geben somit ein Versprechen auf authentische Darstellungen. Sie haben zwar einen realen Hintergrund, stellen aber selbstverständlich eine medienspezifische, dramaturgische Konstruktion dar. Kandidatinnen und Kandidaten werden gezielt auf konflikthafte Polarisierung gecastet, Schnitt und Auswahl der gezeigten Szenen sind bewusst gewählt, um möglichst spannend, emotional und dramatisch zu erzählen. ${ }^{17}$

Dies wird deutlich, als Tony von einem Mitglied der Jury aufgefordert wird, eine besonders heikle Passage vorzutragen. Es handelt sich um die Geschichte eines gewissen Oskar, der bei seiner Flucht in der Schweiz festgenommen und wieder abtransportiert wurde, was aller Wahrscheinlichkeit nach einem Todesurteil gleichkommt. Tony beschränkt sich diesmal nicht nur auf die eigentliche Gedächtnisleistung, sondern versucht zugleich, die Botschaft selber zu deuten, was bei der Jury auf Unverständnis stößt:

TONY Fühlen Sie da nicht wie ihr Blut aus dem Kopf sackt, möchten Sie da nicht auch mit der Faust auf etwas einschlagen, wenn Sie von dieser Ungerechtigkeit hören.

$[\cdots]$

TONY Ich erzähle Ihnen die scheußlichste Geschichte, die einem Menschen widerfahren kann, und Sie antworten ungerührt: Das ist korrekt. Sind Sie überhaupt ein Mensch, schlägt da ein Herz in Ihrer Brust?

$[\cdots]$

GUIDO Das ist tragisch, aber nicht Thema dieser Sendung. ${ }^{18}$

Ziel und Zweck der Sendung sei nämlich, sich dem Geschmack des lachfreudigen Publikums anzupassen. Die Lachkultur soll ungestört weiter laufen, ohne Impulse, die statt des Lachens ein Innehalten, vielleicht sogar eine von Schuldgefühlen gespeiste Zermürbtheit oder gar Weinen herbeirufen könnten. Bei einem solchen Talent-Wettbewerb wird das Lachen eher durch das Komische realisiert, nicht etwa durch den Humor. Mit dem Komischen ist aber diesmal nicht nur das zum Lachen Anregende gemeint. Vielmehr lässt sich der Lacheffekt auf die zweite Bedeutung des Adjektivs komisch zurückführen: Komisch, also eigenartig und sonderbar soll die jeweilige Performance sein, um beim Publikum die erwünschte Reaktion auszulösen. So versucht auch Tony, mit einer lächerlichen Mütze schwierige, ja unange-

17 Margreth Lüneborg and Claudia Töpper, Gezielte Grenzverletzungen - Castingshows und Werteempfinden, https://www.bpb.de/apuz/33554/gezielte-grenzverletzungen-castingshowsund-werteempfinden.

18 Bärfuss, „Zwanzigtausend Seiten“, 188. 
nehme Sachverhalte abzuhandeln. Nicht nur durch Humor, also durch durchdachte sprachliche Handlungen wird das Komische und folglich das Lachen erzeugt, sondern durch Visuelles und Auditives, wo die Vernunft des Menschen keine große Rolle spielt.

Der Satz „Es ist tragisch“, den einer der Juroren ausspricht, kann somit nicht als ernstgemeinte Aussage und ehrliche Empathie verstanden werden, sondern als abgegriffene Wendung, die geheuchelte Anteilnahme formal zum Ausdruck bringt.

Der wohl überraschendste Moment kommt gleich nach diesem Scheitern, als Oskar auftaucht und Tony mitteilt, er habe überlebt:

TONY Ich habe das für Sie getan.

OSKAR Das wäre nicht nötig gewesen.

TONY Und die Erinnerung.

OSKAR Ich habe alles getan, um diese Zeit zu vergessen. Ich will mich nicht erinnern. Und deshalb bin ich in diesem Land. Hier erinnert sich keiner.

TONY Aber das ist doch Unrecht.

OSKAR Soll ich deswegen Trübsal blasen bis ans Ende meiner Tage. Ich finde, ich habe nach der ganzen Geschichte jeden Spaß verdient, der das Leben zu bieten hat.

TONY Aber wozu denn das Ganze, die Erinnerung, die Geschichte, der Kampf um das Gedächtnis.

OSKAR Frag mich das nicht, Tony, frag mich das nicht. Ich habe nie darum gebeten. ${ }^{19}$

Eine noch größere Überraschung als das Überleben dieses Mannes stellt für Tony die Tatsache dar, dass dieser das Weinen im übertragenen Sinne des Wortes (Trübsal blasen) zurückweist und das Positive, ja vielleicht das mit dem Lachen Verbundene (Spaß haben) akzentuiert. Oskar reiht sich also zu den Menschen ein, die diese Aufführung im Fernsehstudio mitverfolgt haben. Ähnlich wie sie verzichtet auch er auf eine Rückbesinnung und zieht es vor, seine unangenehmen Erinnerungen zu verdrängen und stattdessen einfache Unterhaltungen zu genießen.

Betrachtet man die Reaktion von Tony und Wüthrich genauer, bieten sich zwei Herangehensweisen, mit den Ungerechtigkeiten der Vergangenheit, und der ausgebliebenen kritischen Auseinandersetzung der Gesellschaft fertigzuwerden. Entweder sich von der Gemeinschaft loszusagen und weinend außerhalb des öffentlichen Lebens zu verharren, oder sich andersrum vollständig zu integrieren, an den Veranstaltungen, die die Lachkultur fördern sollen, teilzuhaben und quasi unbeschwert und unreflektiert mitzu-

19 Bärfuss, „Zwanzigtausend Seiten“, 191. 
lachen. Weinen kann somit als reflektierende Handlung verstanden werden, Lachen als Strategie, von unangenehmen Sachverhalten Abstand zu halten.

Tony schlägt aber weder den Weg des Lachens noch den des Weinens ein. Er ist kognitiv nicht imstande, die eine oder andere Partei zu ergreifen. Deshalb flüchtet er sich in die Obhut der behandelnden Ärztin. Nach diesem Misserfolg versinkt Tony in Schwermut und lässt sich von Gosbor zu einem Experiment überreden, bei dem ihm erneut ein gewichtiges Paket von Büchern auf den Kopf geworfen werden soll. Diesmal jedoch soll das Paket nicht - so Gosbor - mit „ungefähre[m] Geplauder“20 und „überflüssige[n], nutzlose[n], sogar schädliche[n] Halbinformationen über ein dämmriges Kapitel der jüngeren Zeitgeschichte“21 gefüllt sein, sondern mit „Wissen, das man wahrhaft Wissen nennen darf, weil es nämlich weder der Auslegung, noch der Deutung oder der Interpretation bedarf, weil es sich nämlich nicht auf schöngeistige Tändeleien, sondern auf überprüfbare Fakten stützt" ${ }^{\prime 22}$. Informatik, Materialkunde, Marketing, Chinesisch, italienische Kochrezepte „sämtliche Filme von 1890 bis in der Gegenwart, [...] [p]lus ein bisschen von Goethe und Schiller, zum Abrunden“23 finden Zugang zu seinem Gehirn. Als Resultat dieses Experiments verfügt Tony tatsächlich über alles oben Genannte, ist allerdings nicht mehr fähig, eigene Schlussfolgerungen zu ziehen, und bleibt auf die Fürsorge anderer angewiesen, wissenschaftliche Statements auf Chinesisch vor sich hin plappernd.

\section{Fazit}

Obwohl die Figuren Wüthrich und Tony bis an die Grenzen ihrer Emotionen gegangen sind, ist es ihnen nicht gelungen, die Einstellung ihrer Schweizer Mitbürger zur eigenen Vergangenheit zu verändern. Wüthrichs Weinen sollte dabei nicht bloß als trauriges Weinen betrachtet werden, sondern als verzweifelter Versuch, mit der Schuld der Gemeinschaft zurechtzukommen. Was Lachen anbelangt, ist Tony weder derjenige, der selber lacht, noch ist er jemand, der freiwillig ins Lächerliche gezogen wurde. Er fällt seiner Naivität zum Opfer - der Hoffnung, für die Gesellschaft Gutes tun zu können und darüber hinaus seine eigene psychische Belastung loszuwerden. Er macht sich zum Narren, indem er die Absicht verfolgt, durch eine komische Form, geschichtsträchtige Inhalte zu liefern. Doch in dieser Annahme

\footnotetext{
Bärfuss, „Zwanzigtausend Seiten“, 198.

1 Bärfuss, „Zwanzigtausend Seiten“, 204.

Bärfuss, „Zwanzigtausend Seiten“, 206.

23 Bärfuss, „Zwanzigtausend Seiten“, 202.
} 
täuscht er sich völlig, denn nicht einmal die Begegnung mit einem Überlebenden spendet ihm Trost.

Damit gelange ich zu meiner ersten Forschungsfrage. Obwohl Wüthrich und Tony Weinen und Lachen verkörpern, lässt sich ihre Gegenüberstellung nicht als komplementär bezeichnen. Im ersten Fall dient das Weinen vor allem als biologische Reaktion auf die ausweglose Lage des Betroffenen. Des Weiteren denkt Wüthrich, sich selbst und womöglich auch seine Mitmenschen durch Weinen von der Schuld reinzuwaschen. Zu dieser biologisch bedingten Auffassung des Weinens kommt aber Tonys Lachen hinzu, wobei es nötig ist zu betonen, dass nicht er es ist, der lacht, sondern das Lachen anderer soll die geeignete Plattform für seine Botschaft werden. Hierbei rückt das Lachen nicht nur als körperlicher Vorgang, sondern vielmehr als Medium in den Vordergrund. Weinen und Lachen werden sowohl auf der basalen Triebebene als auch auf einer abstrakten ausgelebt. Obwohl somit eine äußert breite Basis für eine Umstimmung der Öffentlichkeit geschaffen wird, führt allerdings keine davon zum erwünschten Ziel.

Zur zweiten Forschungsfrage lässt sich feststellen, dass der Grund für das Scheitern der beiden Protagonisten sich vorerst als der gleiche bezeichnen lässt. Die Gesellschaft ist nicht einsichtig und lehnt es ab, diesen beiden Männern Gehör zu verschaffen. Abgesehen von ihrem Handeln bleibt der vorherrschende Diskurs gegenüber einer ehrlichen Vergangenheitsbewältigung unverändert. Die Mechanismen, die dabei eine entscheidende Rolle spielen, sind jedoch unterschiedlich. Während Wüthrich (ähnlich etwa wie Prof. Blonay) aus der Gesellschaft ausgeschlossen wurde und abgeschottet von jeglichem Menschenkontakt allmählich in Verzweiflung versinkt, verhält es sich bei Tony anders. Seine Versuche, die Gesellschaft umzustimmen, sind anderweitig motiviert, weshalb auch sein Scheitern anders ausfällt. Er strebt vor allen Dingen nach der Wiedererlangung der eigenen Normalität. Obwohl ihn die Geschichten, die er im Kopf herumträgt, zunehmend persönlich beschäftigen, ist er schließlich bereit, alles zu vergessen und sich in die Reihen der Vergangenheitsverweigerer zu stellen.

Die dritte Frage nach der Rolle des Weinens und Lachens bezüglich des Verhältnisses der Gesellschaft zu Wüthrich und Tony kann folgendermaßen beantwortet werden: Weinen erfüllt die Funktion einer biologischen Auseinandersetzung, während Lachen eine viel komplexere und differenziertere Darstellung erfährt. Sie entfernt sich nämlich von der körperlichen Erfahrbarkeit, indem sie eine gesellschaftliche Form einnimmt. Das Lachen wird zur Lachkultur ausgeführt, die dem allgemein akzeptablen Diskurs untergeordnet ist. Diese erweist sich aber als ungebräuchlich für die Überbringung von unangenehmen Fakten, denen ein brisanter gesellschaftskritischer Charakter innewohnt. 
Die in der Einleitung angesprochene Ausweglosigkeit, die Aufarbeitung eigener Vergangenheit nicht reflektierter und kritischer gestalten zu können, auf die durch Lachen und Weinen in unterschiedlichen oben angedeuteten Ausprägungen reagiert wird, sollte man noch durch eine dritte Option ergänzen: Verzicht. Die Hauptfigur Tony lehnt es ab, mit Wüthrich mitzuweinen oder mit Oskar an der Kultur des Vergessens teilzuhaben. Er wählt den einfachsten Weg, den er auch vor seinem Unfall zu begehen pflegte. Eher als handelnder Geist sich einem unsicheren und womöglich frustrierten Schicksal aussetzend, verzichtet er lieber freiwillig auf seine Gabe, die geschichtliche Vergangenheit reflektiert betrachten zu können und lässt sich abermals bevormunden und andere über ihn entscheiden. Das Ergebnis ist definitiv nicht zum Lachen.

\section{References}

Bärfuss, Lukas. "Zwanzigtausend Seiten.” In Bärfuss, Lukas. Malaga, Parzifal, Zwanzigtausend Seiten. Stücke, 124-206. Göttingen: Wallstein Verlag, 2012.

Brázda, Radim. "Odo Marquard, Humor, Vernunft und Lachen." Pro-Fil 16, no. 2 (2015): 51-68.

Die Schweiz, der Nationalsozialismus und der Zweite Weltkrieg: Schlussbericht, edited by Jean-François Bergier, Wladyslaw Bartoszewski, Saul Friedländer, and Harold James, Zürich 2002. https://www.researchgate.net/publication/278749844_Die_Schweiz_der_Nationalsozialismus_und_der_Zweite_Weltkrieg_Schlussbericht.

Empathie und Erzählung, edited by Claudia Breger, and Fritz Breithaupt. Freiburg i. Br/Berlin/Wien: Romach Litterae, 2010.

Duden. Deutsches Universalwörterbuch. Eigth edition, Berlin: Duden Verlag, 2015.

Fischer, Joachim. "Helmut Plessner: Lachen und Weinen. Eine Untersuchung nach den Grenzen des menschlichen Verhaltens." In Hauptwerke der Emotionssoziologie, edited by Konstanze Senge, and Rainer Schützeichel, 274-279. Wiesbaden: Springer, 2013.

Handlungsmuster der Gegenwart. Beiträge zum Werk von Lukas Bärfuss, edited by Gunreben, Marie and Marx, Friedhelm. Würzburg: Königshausen und Neumann, 2017.

Hentschel, Ingrid. "Spielen mit der Performanz. Transformationen des Komischen im Gegenwarttheater." Maske und Kothurn 54, no. 4 (2005): 214-226. 
Lüneborg, Margreth, and Töpper Claudia. Gezielte Werteverletzungen-Castingshows und Werteempfinden. https://www.bpb.de/apuz/33554/gezielte-grenzverletzungen-castingshows-und-werteempfinden.

Mazenauer, Beat. Elend ist keine Stilfrage. https://nachtkritik.de/index.php?option=com_content\&view=article\&id $=6546$ :zwanzigtausendseiten-ua-lars-ole-walburgs-inszenierung-von-lukas-baerfuss-neuemstueck-am-schauspielhaus-zuerich-fragt-wieviel-verantwortung-wirfuer-die-vergangenheit-tragen-wollen\&catid=38:die-nachtkritik\&Itemid $=40$.

Plessner, Helmut. Lachen und Weinen. Eine Untersuchung der Grenzen menschlichen Verhaltens. München: Leo Lehnen/Francke 1950.

Rasini, Vallori. "Menschlicher Ausdruck und Grenzverwirklichung. Lachen und Weinen." In Expressivität und Stil. Helmuth Plessners Sinnes- und Ausdrucksphilosophie, edited by Bruno Accarino, and Matthias Schlossberger, Internationales Jahrbuch für Philosophische Anthropologie 1 (Berlin: Akademie 2008): 199-208.

Steiger, Claudio. Geschichte ohne Schüler. https://www.nzz.ch/geschichte_ohne_schueler-1.14771452.

Letnansky, Lisa. Wozu das ganze Spektakel? https://www.nahaufnahmen.ch/2012/02/04/lukas-barfuss-zwanzigtausend-seiten-schiffbaubox-zurich/.

Tanja, Thomas. '“Mensch, burnen musst Du!' Castingshows als Werkstatt des neoliberalen Subjekts." Zeitschrift für Politische Psychologie 12, no. 1/2 (2004): 191-208.

\section{Weinen und Lachen als tragende Säulen der Schweiz-Kritik im Theaterstück Zwanzigtausend Seiten von Lukas Bärfuss}

Zusammenfassung: Im Mittelpunkt dieses Beitrags steht das bis jetzt wenig erforschte Theaterstück Zwanzigtausend Seiten des Schweizer Dramatikers Lukas Bärfuss. Die Kritik an der Schweizer Gesellschaft wird unter dem Fokus der emotionalen Erscheinungen Lachen und Weinen dargestellt. Im ersten Schritt wird auf die Begriffe Weinen und Lachen aus biologischer sowie gesellschaftlicher Sicht eingegangen. Im Speziellen wird unterschieden, wie diese Phänomene mit dem Tragischen bzw. dem Komischen (fokussiert auf die emotionalen Äußerungen, die im Weinen und Lachen ihren Ausdruck finden) zusammenhängen und welche Rolle in dieser Wechselbeziehung die Vernunft spielt. Ferner wird auch der Bezug von Weinen und Lachen zur Empathie(losigkeit) der Gemeinschaft gegenüber dem Individuum näher betrachtet und an konkreten Textpassagen festgemacht. Die Leitthese dieser Abhandlung lautet: Man kann seine emotionalen Grenzen beliebig ausweiten, jedoch vermag weder Weinen noch Lachen den Strom des vorherrschenden Diskurses innerhalb einer Gesellschaft zu unterbrechen.

Schüsselwörter: Lachen, Weinen, Zwanzigtausend Seiten, Lukas Bärfuss, Empathie, SchweizKritik. 


\title{
Płacz i śmiech jako podpora krytyki Szwajcarii w sztuce Zwanzigtausend Seiten Lukasa Bärfussa
}

\begin{abstract}
Abstrakt: Przedmiotem analizy są narzędzia krytyki społeczeństwa szwajcarskiego oparte na zjawiskach emocjonalnych - śmiechu i płaczu - zastosowane w słabo dotychczas zbadanej sztuce Zwanzigtausend Seiten szwajcarskiego dramaturga Lukasa Bärfussa. W pierwszym kroku omawiane są pojęcia płaczu i śmiechu z perspektywy biologii i aspektów społecznych. Szczególnie podkreślony został związek tych fenomenów z kategoriami tragizmu i komizmu oraz zdefiniowana została rola rozumu (Vernunft), jaką odgrywa on w tej wzajemnie warunkującej się relacji. Następnie zbadana została na konkretnych fragmentach tekstu relacja płaczu i śmiechu do empatii (lub jej braku) we wspólnocie wobec jednostki. Zgodnie z tezą artykułu, można dowolnie poszerzać własne granice emocjonalne, lecz ani płaczem ani śmiechem nie da się przerwać prądu dominującego dyskursu w społeczeństwie.
\end{abstract}

Słowa kluczowe: śmiech, płacz, Zwanzigtausend Seiten, Lukas Bärfuss, empatia, krytyka Szwajcarii. 